\title{
Prevalence of heart disease in a population of domestic cats in Poland: A retrospective study
}

\author{
MARTA PARZENIECKA-JAWORSKA, MAGDALENA GARNCARZ \\ Department of Pathology and Veterinary Diagnostics, Faculty of Veterinary Medicine, \\ Warsaw University of Life Sciences, Nowoursynowska 159, 02-776, Warsaw
}

Parzeniecka-Jaworska M., Garncarz M.

\section{Prevalence of heart disease in a population of domestic cats in Poland: A retrospective study}

\section{Summary}

The survey included 514 cats brought to the Department of Small Animals within 5 years. The cats were examined cardiologically (clinical examination, echocardiography and electrocardiographic examination) and then divided into cats with cardiac changes and cats with problems other than heart disease. The study was designed to show the most common cardiac disease in cats and the breeds represented. The study found that the most prevalent cardiac diseases in cats were cardiomyopathies, which were found in $16 \%$ of all cats examined, while congenital malformations were found in $8 \%$ of the cats. Cats of 19 different breeds were represented, most of which were European Shorthair cats and Maine Coons. The European Shorthair breed had the largest number of healthy cats $\mathbf{( 4 0 . 9 \%}$ of all healthy cats) as well as the largest number of cats with congenital heart disease (35.9\% of all cats with congenital heart disease) and the largest number of cats with cardiomyopathies $\mathbf{5 0 . 6 \%}$ of all cats with cardiomyopathies).

Keywords: feline, heart diseases, cardiomyopathies, congenital heart diseases

Cats can be affected by acquired heart disease or, less frequently, congenital heart defects. It is estimated that congenital malformations account for about $5 \%$ of heart problems in this species $(9,16)$. Congenital heart defects in cats include ventricular septal defect (VSD), patent ductus arteriosus (PDA), subaortic stenosis (SAS), tetralogy of Fallot (a combination of pulmonary artery stenosis, intraventricular septal defect, right ventricular hypertrophy and an overriding aorta), dysplasia of the mitral or tricuspid valve, and diaphragmatic pericardial hernia. No breed predisposition has been reported for these defects $(1,8,13,15)$. Acquired diseases are much more common in cats. Among them, we can distinguish cardiomyopathies, which are myocardial diseases affecting the myocardial tissue. Most cardiomyopathies in cats are primary and idiopathic - only the etiology of dilated cardiomyopathy (DCM) has been identified and proven to be secondary to taurine deficiency. The most common cardiac disease in cats is hypertrophic cardiomyopathy (HCM), in the course of which a concentric hypertrophy of left ventricular walls and/or papillary muscles occurs. A predominant autosomal character of inheritance has been proven in some breeds of cats, namely the Maine Coon and Ragdoll $(10,17)$. However, a predominance is recognized not only in the Maine Coon and Ragdoll, but also in the Persian, Bengal, American Shorthair, British Shorthair, Norwegian Forest and Cornish Rex cats (6). This disease can occur in any cat breed, including European cats. Another cardiomyopathy that occurs in cats is restrictive cardiomyopathy (RCM). In this disease, there is limited diastolic filling of the ventricles. No breed predisposition to this disease has been described. All cases which do not include changes specific for HCM, RCM or DCM are termed unclassified cardiomyopathies (UCM).

The purpose of this study was to show the frequency of heart disease in cats in Poland, as well as to determine which cat breeds are the most frequent cardiac patients and which heart diseases are detected most often in particular cat breeds.

\section{Material and methods}

Information from the first visit charts of 6557 feline patients was extracted and analyzed. These cats were examined between 2012 and 2016 by one of the two cardiologists of the Faculty of Veterinary Medicine, Warsaw University of Life Sciences. Out of the 6557 cats, 514 (7.8\%) were examined cardiologically. They comprised 279 males and 235 females, aged from 1 to 244 months. The cats were examined for various reasons, some as a prophylactic measure (because of breed predisposition to heart 
disease or prior to surgery), others due to disturbing clinical symptoms. Among the most commonly reported symptoms was dyspnoea, especially after exercise, and rapid fatigue during play. Some patients were referred for cardiologic examination because of ocular problems and suspicion of hypertension. All patients underwent a full clinical examination, including measurement of temperature and the respiratory rate per minute, mucous membrane, lymph node and pulse assessment, as well as heart and lung auscultations. Subsequently, depending on the changes observed, echocardiography, electrocardiogram and/or blood pressure measurements were performed by the Doppler method. This study complies with national and institutional guidelines on the use of animals in clinical research according to the Polish legal act of January 21, 2005, concerning experiments performed on client-owned animals. On the basis of these examinations, the cats were divided into patients with heart disorders (201 animals) and patients with extracardiac problems (313 animals). Among the patients with heart disease, there were cats with congenital heart diseases (39 animals), cardiomyopathies ( 83 animals), myocardial hypertrophy (57 animals) and unspecific cardiac changes (22 animals). The remaining animals included cats without heart disease (237 animals) and cats with systemic hypertension (57 animals) or pleural effusion (19 animals). Left ventricular hypertrophy patients without left atrial enlargement were placed in the group of hypertrophic cats. Cats were diagnosed with hypertrophic cardiomyopathy if echocardiography revealed left ventricular and/or papillary muscle hypertrophy and left atrial enlargement after secondary causes of hypertrophy had been excluded. A separate group was established for cats with unspecific cardiac changes in echocardiography that did not produce clinical changes and did not cause cardiac enlargement, for example, mitral regurgitation and arrhythmias.

\section{Results and discussion}

The animals were divided into 2 groups: those with heart disorders (i.e. congenital heart diseases, cardiomyopathies, myocardial hypertrophy or unspecific cardiac changes) and those with extracardiac problems (i.e. without heart disorders, with systemic hypertension or with pleural effusion) (Fig. 1). The most frequent congenital heart defect in cats was the ventricular septal defect (Tab. 1). Four cats had multiple congenital defects simultaneously. Two cats had a ventricular septal defect (VSD) and pulmonary stenosis (PS), one cat had pulmonary stenosis and subaortic stenosis (SAS), and one had a VSD and mitral valve dysplasia. Among cats with cardiomyopathies, the majority had hypertrophic cardiomyopathy $(\mathrm{n}=46)$, accounting for $55.5 \%$ of all cats with cardiomyopathies. The second most common acquired disease was restrictive cardiomyopathy, which was found in 27 cats, accounting for $32.5 \%$ of all cardiomyopathies. Unclassified cardiomyopathy occurred significantly less frequently $(\mathrm{n}=8)$, in $9.6 \%$ of cats with cardiomyopathies, whereas dilated cardiomyopathy was rare $(n=2)$, making up only $2.4 \%$ of all cats with cardiomyopathies. All car-

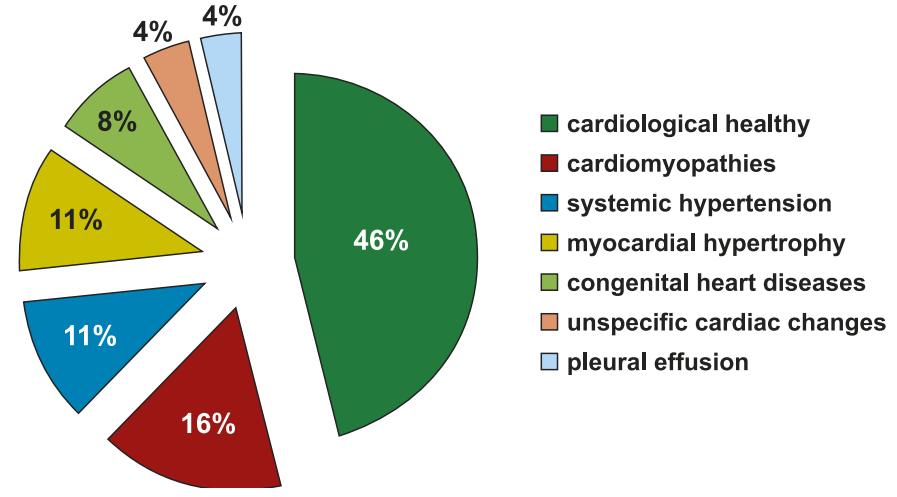

Fig. 1. Division of cats according to cardiac changes

Tab. 1. Congenital heart defects in cats

\begin{tabular}{|l|c|c|}
\hline \multicolumn{1}{|c|}{$\begin{array}{c}\text { Congenital heart } \\
\text { defect }\end{array}$} & $\begin{array}{c}\text { Number of animals } \\
(\mathrm{n})\end{array}$ & $\begin{array}{c}\text { Percentage of animals with } \\
\text { congenital heart defects }\end{array}$ \\
\hline MD or TD & 3 & 7.7 \\
SAS & 6 & 15.4 \\
Diaphragmatic \\
pericardial hernia
\end{tabular}

Explanations: $\mathrm{MD}$ - mitral valve dysplasia, TD - tricuspid valve dysplasia, SAS - subaortic stenosis, PDA - patent ductus artriosus, VSD - ventricular septal defect, PS - pulmonic stenosis

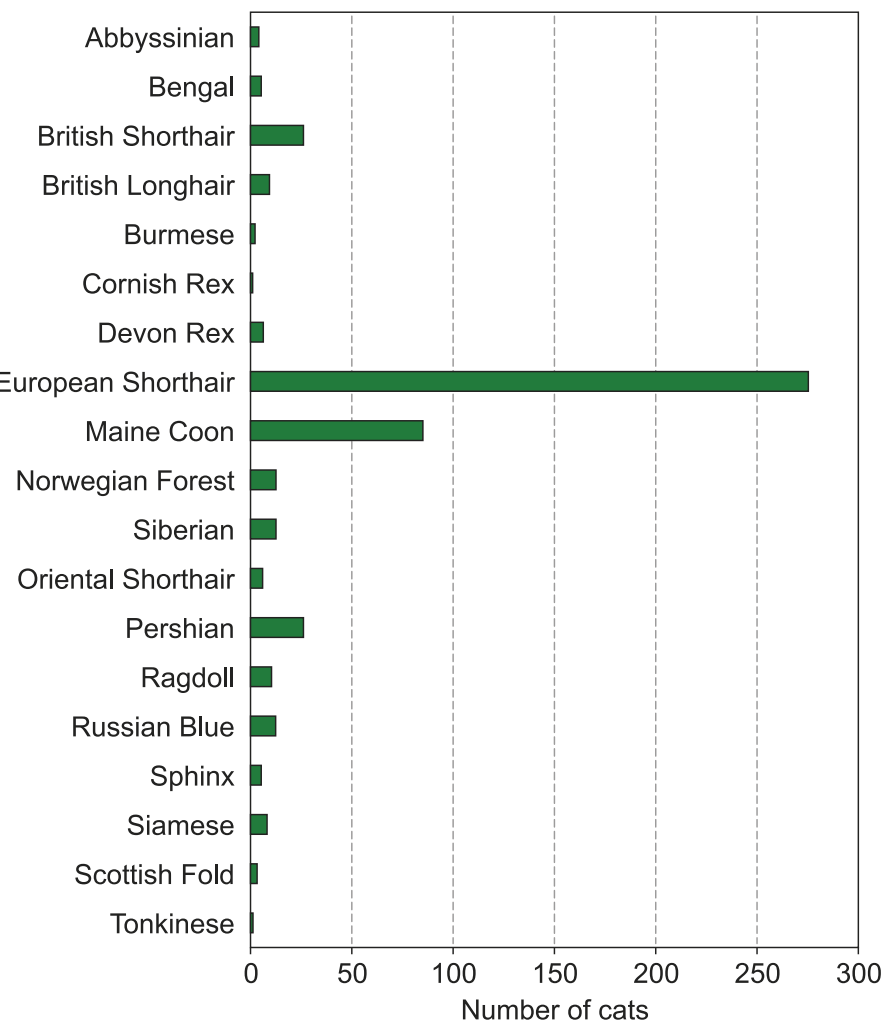

Fig. 2. Division of cats according to breed 
diomyopathies together accounted for most of the heart diseases.

The study also aimed to demonstrate which cat breeds were examined cardiologically most often. There were 19 cat breeds represented in the study, with European Shorthair cats $(n=275)$ being the largest group, accounting for $53.5 \%$ of all tested cats. Maine Coons $(n=85)$ were the second largest group, accounting for $16.5 \%$ of all tested cats (Fig. 2).

The study also determined which cat breeds predominated in the cardiologically healthy group, in the group with congenital malformations, and in the group with cardiomyopathies (Tab. 2). The two most numerous cat breeds in the healthy group were the European breed $(\mathrm{n}=97,41 \%$ of all healthy cats) and the Maine Coon breed $(n=53,22.4 \%)$. Congenital defects also occurred most often in the European breed ( $n=14,36 \%$ of all cats with congenital defects). Cardiomyopathies were most often recognized in the European breed $(n=42,50.6 \%$ of all cats with cardiomyopathies), followed by Maine Coon cats $(\mathrm{n}=10,12 \%)$ and Persians $(\mathrm{n}=10$, $12 \%$ ). The breeds with the least percentage of healthy cats included the Persian $(5 / 26,19.2 \%)$, followed by the British Shorthair (12/26, 46.1\%).

Cats are complicated patients because of the difficulty of recognizing clinical signs. Usually no signs are noted by the owner. When a sign is noted, it is most often dyspnoea. In animals with dyspnoea, it is often difficult to diagnose the primary heart condition because changes observed in echocardiography are ambiguous or not typical of a specific heart disease. This may be due to the time of the first examination relative to the clinical course of disease (too early or too late), as one may see minimal unspecific changes or changes secondary to another, non-cardiac disease (e.g. a "burned-out" left ventricle giving an unspecific appearance of the left ventricle in very late stages of HCM, or hypertrophy secondary to systemic hypertension). Therefore, in this study a separate group was created of cats with unspecific cardiac changes of unknown origin. These were mostly regurgitation of atrioventricular valves without visible leaflet changes that did not result in chamber enlargement and/or arrhythmias at the time of study. These cats did not show other echocardiographic changes, specifically hypertrophy or increased echogenicity. A group of cats with non-cardiogenic pleural fluid was also created because of their relatively large number $(4 \%$ of the examined animals). Such cats are often referred to a cardiologist because of their dyspnoea.

Congenital heart disease was recognized in $8 \%$ of the cats. This is in line with other reports found in the literature, citing from 5\% (16) up to 12\% in European cat populations $(9,14,16,19)$. According to these studies, the most common congenital heart disease is
Tab. 2 The numbers of cats with cardiomyopathies, cats with congenial heart diseases and cardiologically healthy cats of particular breeds

\begin{tabular}{|c|c|c|c|}
\hline Breed & $\begin{array}{l}\text { Cardiomyopathies } \\
\text { (n) }\end{array}$ & $\begin{array}{l}\text { Congenital heart } \\
\text { disorders (n) }\end{array}$ & $\begin{array}{l}\text { Cardiologically } \\
\text { healthy (n) }\end{array}$ \\
\hline Abyssinian & 0 & 1 & 2 \\
\hline Bengal & 0 & 2 & 3 \\
\hline British Shorthair & 5 & 3 & 12 \\
\hline British Longhair & 3 & 0 & 4 \\
\hline Burmese & 0 & 0 & 2 \\
\hline Cornish Rex & 0 & 0 & 1 \\
\hline Devon Rex & 1 & 1 & 2 \\
\hline European Shorthair & 42 & 14 & 97 \\
\hline Maine Coon & 10 & 9 & 53 \\
\hline Norwegian Forest & 4 & 1 & 3 \\
\hline Siberian & 1 & 0 & 10 \\
\hline Oriental Shorthair & 1 & 2 & 1 \\
\hline Persian & 10 & 2 & 5 \\
\hline Ragdoll & 0 & 0 & 10 \\
\hline Russian Blue & 1 & 2 & 8 \\
\hline Sphinx & 1 & 2 & 1 \\
\hline Siamese & 1 & 0 & 4 \\
\hline Scottish Fold & 0 & 0 & 3 \\
\hline Tonkinese & 0 & 0 & 1 \\
\hline
\end{tabular}

VSD, and this was confirmed by our study. The second most common defect in this study was subaortic stenosis and a combination of defects. Another frequent congenital heart defect in cats reported in the literature is dysplasia of the mitral and tricuspid valves, which in our study was relatively rare $(15,16)$. Most of the cats in our study were adult or geriatric, and therefore cases of atrio-ventricular dysplasia were not diagnosed, because the cats never displayed clinical signs. Prophylactic examinations for congenital heart defects are not routinely performed in Poland, and therefore the breeds predisposed to this disease are not screened. There is also the possibility that adult cats with mitral or tricuspid valve regurgitation and no other lesions may have dysplastic changes on these valves.

The most commonly diagnosed acquired heart disease in this study was hypertrophic cardiomyopathy. A similar situation is reported in the literature, in which it is estimated to account for two thirds of all cardiomyopathy cases in cats $(2,5,7)$. A separate group of cats with myocardial and/or papillary muscle hypertrophy seen in echocardiography was established in the study during the first visit in cardiological office. Due to the lack of more severe lesions (including left atrial enlargement) and the lack of information about secondary hypertrophy (hypertension, hyperthyroidism), it is impossible to diagnose primary idiopathic hypertrophic cardiomyopathy (5). However, there is a high probability that most of these cats will develop this disease. These patients did not show signs typical of hypertensive diseases, but a recheck pressure 
measurement was recommended. A high percentage of restricted cardiomyopathy was reported in this study, which has been described in the literature as a relatively rare disease (8). It is important to note that in the present study the diagnosis of restricted cardiomyopathy in cats was based on a reduced diastolic volume of one or both ventricles with abnormal diastolic inflow, normal to slightly decreased systolic function and normal wall thickness (9). However, in cats, the exact diagnosis of any cardiomyopathy, particularly the restrictive form with myocardial fibrosis, is possible only by histopathological examination. Therefore, it is difficult to clearly classify such cases as RCM or UCM.

The group of hypertensive cats included individuals with primary or secondary hypertension $(4,12)$. Secondary hypertension was usually related to renal failure, less often to hyperthyroidism. The study shows that this is a frequent condition in older cats (mean age: 170 months). Most cats in the hypertensive group had advanced hypertension with secondary changes, such as myocardial hypertrophy or ophthalmic changes.

European Shorthair cats were the largest group in the present study, most likely because they are the most numerous breed in Poland. The second most common breed is the Maine Coon (11). Because of the Maine Coon's popularity and the fact that breeders are aware of their predisposition to hypertrophic cardiomyopathy $(10,17)$, the Maine Coon is often screened for heart disease and may be overrepresented. Other breeds predisposed to this disease are the Ragdoll and the British Shorthair (11). The breed most frequently affected by heart disease is the Persian. As many as $69 \%$ of Persian cats had various forms of cardiomyopathy, congenital malformations or unclassified cardiac changes, while only $19.2 \%$ were healthy. The rest of the Persian cats $(11.8 \%)$ had hypertension or fluid in the pleural cavity. It appears that this breed is laden with various diseases and would benefit from additional studies on the exact types and frequency of heart disease. On the other hand, this might result from the lack of owner awareness and very few prophylactic tests in this breed. Cardiomyopathies, occurring in $38.5 \%$ of Persian cats, were the most frequently noted abnormality. Most of those $(8 / 10)$ were HCM, strongly suggesting a predisposition to HCM in this breed. We know that Persian cats are predisposed to hypertrophic cardiomyopathy $(6,8,9)$, but they are not listed as the most common breed for this disease. Severe cases of hypertrophic cardiomyopathy in Persian cats have been reported (18), as have congenital defects, such as tetralogy of Fallot (3). However, the increased susceptibility of Persian cats to heart disease has not been described.

The study showed the frequency of particular heart diseases in cats and determined which breeds were most commonly affected. Congenital heart defects accounted for $8 \%$ of all cases in this study, and the most common among them was the ventricular septal defect. Such results support the general trend described in the literature. Among acquired diseases, hypertrophic cardiomyopathy was the most prevalent, which also concurs with other studies. The study examined 19 breeds of cats, among which the largest population were European cats. This breed was dominant among healthy cats as well as cats with congenital heart defects and cardiomyopathies. Maine Coons were the second largest group. Healthy cats of this breed constituted the second largest population of healthy cats but a significant number of Maine Coons suffered from cardiomyopathies. Persian cats proved to be the most frequently affected breed, with the number of affected animals exceeding the number of healthy ones. Hypertrophic cardiomyopathy was the predominant heart disease in this breed.

\section{References}

1. Bascuñản A., Thieman Mankin K. M., Saunders A. B., Bright J. M., Scharf V., Singh A., O'Sullivan L., Brisson B., Estrada A. H., Tou S. P., Ruoff C.: Patent ductus arteriosus in cats (Felis catus): 50 cases (2000-2015). J. Vet. Cardiol. 2017, 19, 35-43.

2.Bishop S. P.: Canine and feline cardiomyopathy. Proc. North Am. Vet. Commun., Orlando, Florida 2006.

3. Choi W.-J., Suh S., Choi R., Hyun Ch.: Acyanotic tetralogy of Fallot in a Persian cat. Can. Vet. J. 2016, 57, 596-600.

4. Chung-Hui L., Chun-Ju Y., Yu-Hsin L., Hui-Pi H.: Systolic blood pressure of clinically normal and conscious cats determined by an indirect Doppler method in a clinical setting. J. Vet. Med. Sci. 2006, 68, 827-832.

5. Ferasin L.: Feline myocardial disease, 1: Classification pathophysiology and clinical presentation. J. Feline Med. Surg. Clin. Pract. 2009, 3-11.

6. Fuentes V. L., Johnson L., Dennis S.: BSAVA Manual of Canine and Feline Cardiorespiratory Medicine, Brit. Small. Anim. Vet. Assoc. England 2012, 220-237.

7. Häggström J.: Screening for hypertrophic cardiomyopathy in cats. J. Vet. Cardiol. 2015, 17, 134-149.

8. Kienle R. D.: Feline cardiomyopathy, [in:], Tilley L. P., Smith F. W. K., Oyama M., Sleeper M. M.: Manual of Canine and Feline Cardiology. Sounders, St. Louis 2008, 151-175.

9. Kittleson M. D.: Hypertrophic cardiomyopathy, [in:] Kittleson M. D., Kienle R. D. (eds): Small Animal Cardiovascular Medicine. Mosby, Inc., St. Louis, MO 1998, p. 361.

10. Lefebvre S.: Risks and trends in cardiomyopathy. Vet. Focus 2012, 22, 24-25.

11. Parzeniecka-Jaworska M.: Ocena badania echokardiograficznego oraz wybranych parametrów laboratoryjnych jako markerów sercowych we wczesnej diagnostyce kardiomiopatii przerostowej u kotów rasy Maine Coon. Praca dokt., Wydz. Med. Wet. SGGW, Warszawa 2013.

12. Payne J. R., Brodbelt D. C., Luis Fuentes V.: Blood pressure measurements in 780 apparently healthy cats. J. Vet. Intern. Med. 2017, 31, 15-21.

13. Peck C. M., Nielsen L. K., Quinn R. L., Laste N. J., Price L. L.: Retrospective evaluation of the incidence and prognostic significance of spontaneous echocardiographic contrast in relation to cardiac disease and congestive heart failure in cats: 725 cases (2006-2011). J. Vet. Emerg. Crit. Care 2016, 26, 704-712.

14. Scansen B. A., Schneider M., Bonagura J. D.: Sequential segmental classification of feline congenital heart disease. J. Vet. Cardiol. 2015, 17, 10-52.

15. Schrope D. P.: Prevalence of congenital heart disease in 76,301 mixed-breed dogs and 57,025 mixed-breed cats. J. Vet. Cardiol. 2015, 17, 192-202.

16. Tidholm A., Ljungvall I., Michal J., Häggström J., Höglung K.: Congenital heart defects in cats: a retrospective study of 162 cats (1996-2013). J. Vet. Cardiol. 2015, 17, 215-219.

17. Trehion-Sechi E., Tissier R., Gouni V., Misbach C., Petit A. M. P., Bolouka D., Sampedrano C. C., Castaignet M., Pouchelon J. L., Chetboul V:: Comparative echocardiographic and clinical features of hypertrophic cardiomyopathy in 5 breeds of cats: A retrospective analysis of 344 cases (2001-2011). J. Vet. Intern. Med. 2012, 26, 532-541.

18. White A. J. M.: End-stage hypertrophic cardiomyopathy in a cat. Can. Vet. J. 2015, 56, 509-511.

19. Wustefeld-Janssens B. G., Burrow R., Mõtsküla P., Martin M., DukesMcEwan J.: Clinical findings and treatment outcomes for cats diagnosed with patent ductus arteriosus in the UK: a retrospective study of 19 cases (2004-2012). Vet. Rec. 2016, 179, 17-22.

Corresponding author: Marta Parzeniecka-Jaworska, DVM, PhD, Nowoursynowska 159, 02-766 Warsaw, Poland; e-mail: marta_parzeniecka_ jaworska@sggw.pl 